\title{
Sensibilidad de diferentes clones de Populus spp. al taladro Parantrhene tabaniformis (Rott.) (Lepidoptera, Sesiidae)
}

\author{
Monreal, J.A.* y Selva, M. \\ Departamento de Producción Vegetal y Tecnología Agraria. Escuela Técnica Superior de Ingenieros Agrónomos \\ y de Montes. Universidad de Castilla-La Mancha. Campus Universitario s/n. 02071-Albacete (España).
}

*Autor para correspondencia: Jose.Monreal@uclm.es

\section{Resumen}

Durante el año 2010 se realizó, en la provincia de Albacete, un ensayo con el objetivo de analizar la adaptación y producción de biomasa de diversas especies leñosas interesantes para su utilización como cultivos energéticos. Para dicho ensayo se eligieron, entre otras especies, cuatro clones de chopo. Posteriormente en el año 2013 tras finalizar el ensayo, se procedió a aclarar y podar los chopos desde densidades propias de un aprovechamiento energético (entre 7000 y 10000 pies/ha) hasta densidades normales para producción de madera para desenrollo (350 a 400 pies/ha). Ese mismo año aparece un fuerte ataque de Parantrhene tabaniformis (Rott.), afectando tanto a los rebrotes como a los árboles en pie. De los estudios realizados en este trabajo se ha comprobado la existencia de diferencias significativas en la sensibilidad de los clones de chopo al ataque de este taladro, observándose sobre todo una menor sensibilidad del clon " $V i$ riato" con respecto a los otros tres.

Palabras clave: sensibilidad clonal, oruga perforadora, chopos. 


\section{Introducción}

Actualmente, tanto la Unión Europea como España tienen una gran dependencia energética del exterior, por esto entre las políticas Europeas prioritarias figura el incremento en el empleo de fuentes de energía renovables, que garanticen el suministro, que sean competitivas y que respeten el medio ambiente.

Dentro de esta política destaca la publicación de la Directiva 2009/28/CE relativa al fomento del uso de la energía procedente de fuentes renovables, el Plan de Acción Nacional de Energías Renovables 2011-2020 del gobierno Español, y en nuestro caso la Orden 02/03/2012 de la Consejería de Agricultura de Castilla-La Mancha por la que se establecen ayudas a la producción de cultivos forestales para la obtención de biomasa.

Entre los cultivos energéticos lignocelulósicos las especies de los géneros Populus y Salix son de las más utilizadas en todo el mundo por contar con una amplia selección de variedades mejoradas genéticamente. En el sur de Europa, el chopo es el que presenta un mayor potencial, debido a las condiciones ecológicas de la Península Ibérica (Sixto et al., 2007).

Pero el cultivo del chopo a altas densidades presenta también ciertos problemas, como son la salinidad, los taladros, la competencia con las malas hierbas y la gestión del riego (Baraza, 2013; Sixto, 2010).

Siguiendo en esta línea, durante el año 2010 se realizó, en la provincia de Albacete, un ensayo con el objetivo de analizar la adaptación y producción de biomasa de diversas especies leñosas interesantes para su utilización como cultivos energéticos. Para dicho ensayo se eligieron, entre otras especies, cuatro clones de chopo: Populus trichocarpa $x$ deltoides clon "Beaupré"; Populus trichocarpa $x$ deltoides clon "Raspalje"; Populus trichocarpa $x$ deltoides clon "Unal" y Populus deltoides clon "Viriato" (Sixto et al., 2013).

Posteriormente en el año 2013 tras finalizar el ensayo, se procedió a aclarar y podar los chopos desde densidades propias de un aprovechamiento energético (entre 7000 y $10000 \mathrm{pies} / \mathrm{ha}$ ) hasta densidades normales para producción de madera para desenrollo (350 a 400 pies/ha). Ese mismo año aparece un fuerte ataque de Parantrhene tabaniformis (Rott.), afectando tanto a los rebrotes como a los árboles en pie. En este trabajo se ha realizado un seguimiento de la sensibilidad de los distintos clones de chopo a la clorosis férrica y a dicho taladro. Este taladro tiene predilección al hacer la puesta por las grietas de la madera y heridas de poda y puede producir depreciación de la madera, menor crecimiento y vigor, rotura por el viento, y favorecer la entrada de enfermedades. Ante estos posibles problemas es interesante conocer que clones tienen una mayor resistencia al taladro (Gutierrez y Estirado, 1978). Es probable que la gran cantidad de nuevos brotes delgados y las heridas de poda hayan tenido una influencia importante en el ataque de Paranthrene tabaniformis. 


\section{Material y métodos}

\subsection{Zona de estudio}

La parcela donde se ha realizado el estudio es de propiedad particular, situada en el paraje de "La Chericoca", tiene una extensión de 4,5 ha y se encuentra en el término municipal de Albacete a unos $25 \mathrm{~km}$ de la ciudad. Se trata de una zona eminentemente agrícola rodeada por grandes extensiones de cereales y cultivos herbáceos tradicionales (trigo, maíz, cebada, colza, ajo, cebolla, etc.), en cuanto a leñosas destacan algunas parcelas de almendro. La zona se encuentra situada al sureste de la Meseta Central a una altitud de $719 \mathrm{~m}$ y la pendiente es prácticamente nula. La parcela dispone de sistema de riego localizado por goteo.

\subsection{Diseño de la experiencia}

El diseño experimental inicial partía de una zona de estudio de 4,5 ha con una estructura de parcelas de bloques completos al azar con tres repeticiones con las siguientes características:

— Número de clones: 4 clones

— Densidad: entre 7000 plantas/ha y 10000 plantas/ha

- Turno: 2 turnos (2 y 3 años)

- Método de aprovechamiento: cortas a hecho

- Cuatro clones de chopo: Populus trichocarpa x deltoides clon "Beaupré"; Populus trichocarpa $x$ deltoides clon "Raspalje"; Populus trichocarpa $x$ deltoides clon "Unal" y Populus deltoides clon "Viriato".

Durante el año 2012, al inicio del ciclo vegetativo y comprobándose la existencia de amarilleo en las hojas, se realizó una estimación visual de las copas para valorar que clones se veían más afectados por la clorosis férrica. Para ello se seleccionaron 20 árboles de cada clon y un solo observador estimó, en porcentajes del 5\%, el grado de afección, calculándose la media por clon.

Actualmente y tras la finalización del ensayo de biomasa, en 2013 la parcela tiene unas densidades de entre 350 y 400 pies/ha, y por tanto no mantiene la estructura de parcelas y bloques del diseño original.

En 2015 se seleccionó para este estudio una zona central, con una superficie de unas 2 ha, para evitar el efecto borde. En dicha parcela se han llevado a cabo las siguientes tareas:

- Selección al azar en 2015 de 100 árboles para el estudio ( 25 pies/clon).

- Seguimiento del vuelo de los adultos machos con la colocación de 40 trampas con feromona (50\% delta y $50 \%$ polilleros) a tres metros de altura (Moraal, 1993) y 10 trampas de cada modelo por ha.

- Marcado de los clones con distintos colores: verde (Beaupre), rojo (Raspalje), amarillo (Unal) y blanco (Viriato).

- Conteo del $\mathrm{n}^{\mathrm{o}}$ de heridas/árbol con los siguientes criterios: 


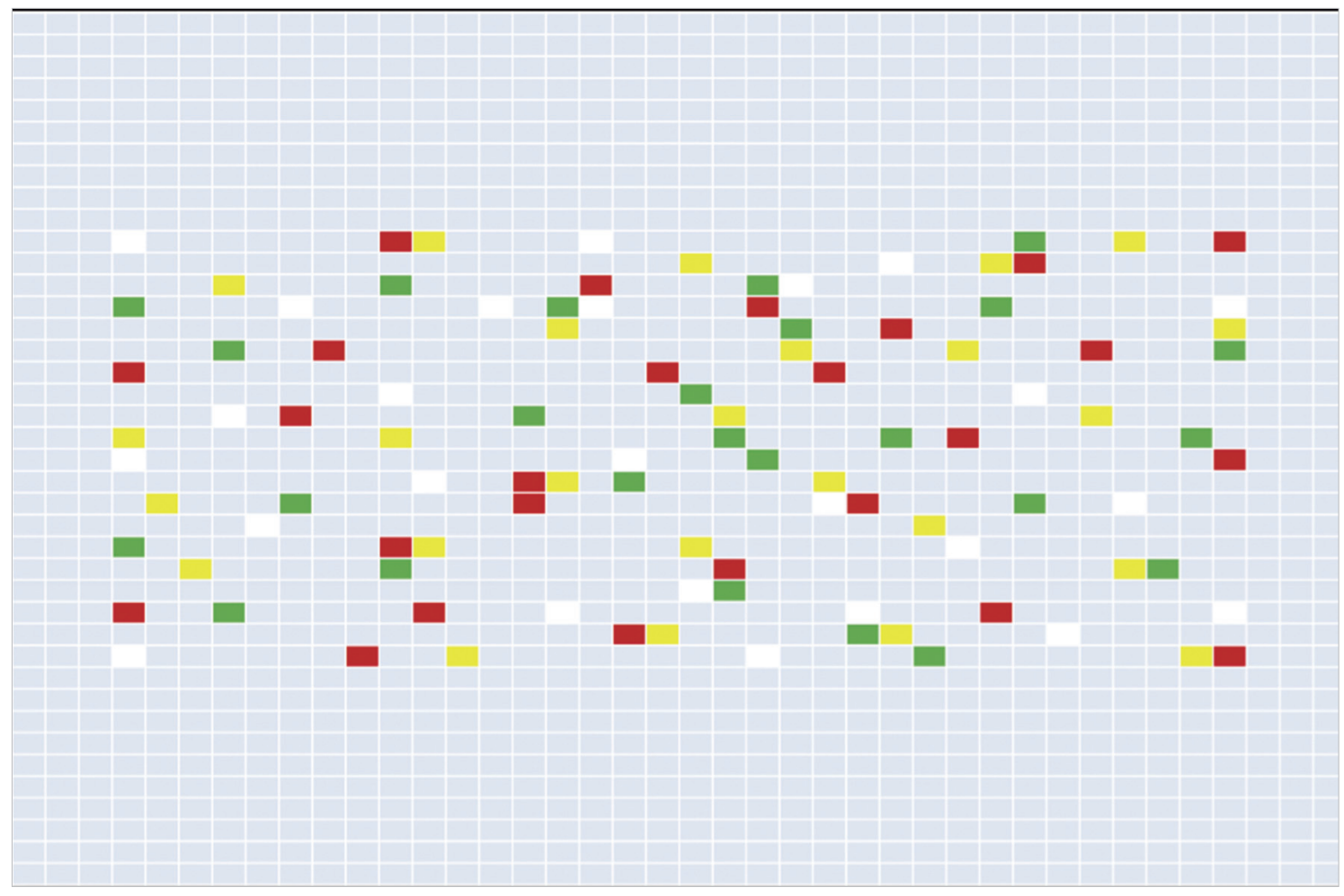

Figura 1. Diseño experimental mostrando la distribución al azar de los árboles en la parcela: verde (Beaupre), rojo (Raspalje), amarillo (Unal) y blanco (Viriato).

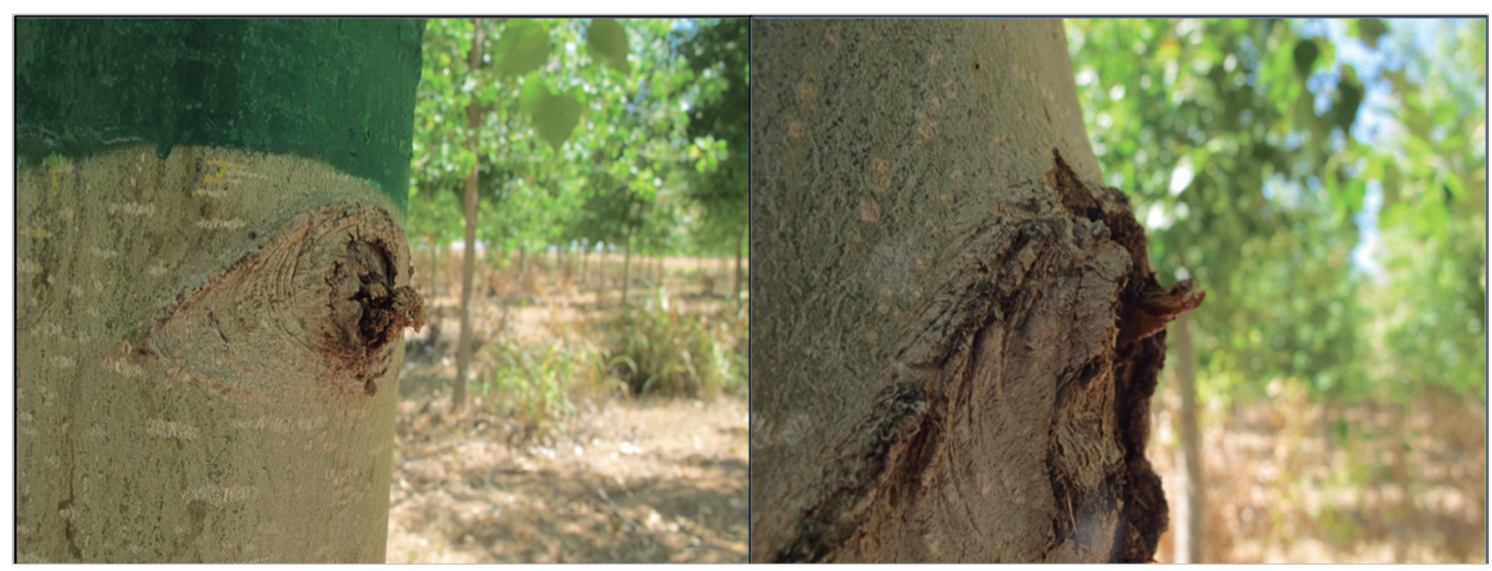

Figura 2. Restos de serrín y de crisálidas de Paranthrene tabaniformis.

- Restos de serrín y sabia en las galerías.

- Restos de exuvios de las crisálidas.

\section{Resultados y discusión}

De la estimación visual de la clorosis férrica de las copas se observaron, como 
Tabla 1. Sensibilidad de los diferentes clones a la clorosis férrica

\begin{tabular}{cccc}
\hline \multicolumn{4}{c}{$\%$ medio de afectación (media \pm d.e.) } \\
\hline Viriato & Beaupre & Unal & Raspalje \\
\hline $8 \pm 2,5$ & $32 \pm 9,2$ & $52 \pm 11,1$ & $36 \pm 5,7$ \\
\hline
\end{tabular}

se muestra en la Tabla 1, diferencias entre clones, resultando el menos afectado y con una mejor recuperación el clon "Viriato".

Para conocer el nivel de población de Paranthrene tabaniformis se colocaron en el mes de mayo dos tipos de trampas con feromona sexual (delta y polilleros), con una densidad de 10 trampas/ha de cada modelo, y se mantuvieron hasta primeros de agosto. Los resultados muestran que la trampa polillero no es útil para la captura del taladro, dado que no se capturo ningún adulto, mientras que se recogieron en las trampas delta una media de $0,85 \pm 0,91$ polillas/trampa/día.

Tabla 2. Número de heridas producidas por el taladro Paranthrene tabaniformis por/clon

\begin{tabular}{cccc}
\hline \multicolumn{4}{c}{ Media $\mathrm{N}^{\text {o }}$ de heridas/clon (media \pm d.e.) } \\
\hline Viriato & Beaupre & Unal & Raspalje \\
\hline $0,4 \pm 0,6$ & $3,8 \pm 2,3$ & $4,9 \pm 2,4$ & $4,4 \pm 2,0$ \\
\hline
\end{tabular}

En cuanto a los daños producidos por el taladro se contabilizó el número de heridas que presentaba cada árbol, resultando el menos dañado el clon "Viriato" en comparación con los otros tres (Tabla 2).

Para el análisis estadístico se ha realizado un ANOVA simple, siendo la variable dependiente el número de heridas y el factor el tipo de clon estudiado. Los resultados muestran con un nivel de significación del $95 \%$ de probabilidad, que hay diferencias significativas entre el clon "Viriato" y los otros tres (Figura 3). Según el test de rangos múltiples para el número de heridas por clon $(\mathrm{n}=100)$, los clones Unal, Raspalje y Beaupre quedarían dentro del mismo grupo homogéneo y estadísticamente diferenciados del clon Viriato.

Tabla 3. ANOVA simple para el número de heridas por clon ( $\mathrm{n}=100)$.

ANOVA simple para el número de heridas por clon $(\mathrm{n}=100)$

\begin{tabular}{cccc}
\hline Fuente & Gl & F-Ratio & P-Value \\
\hline Entre grupos & 3 & 27,24 & $\mathbf{0 , 0 0 1}$ \\
\hline Dentro de grupos & 96 & & \\
\hline Total (Corr.) & 99 & & \\
\hline
\end{tabular}




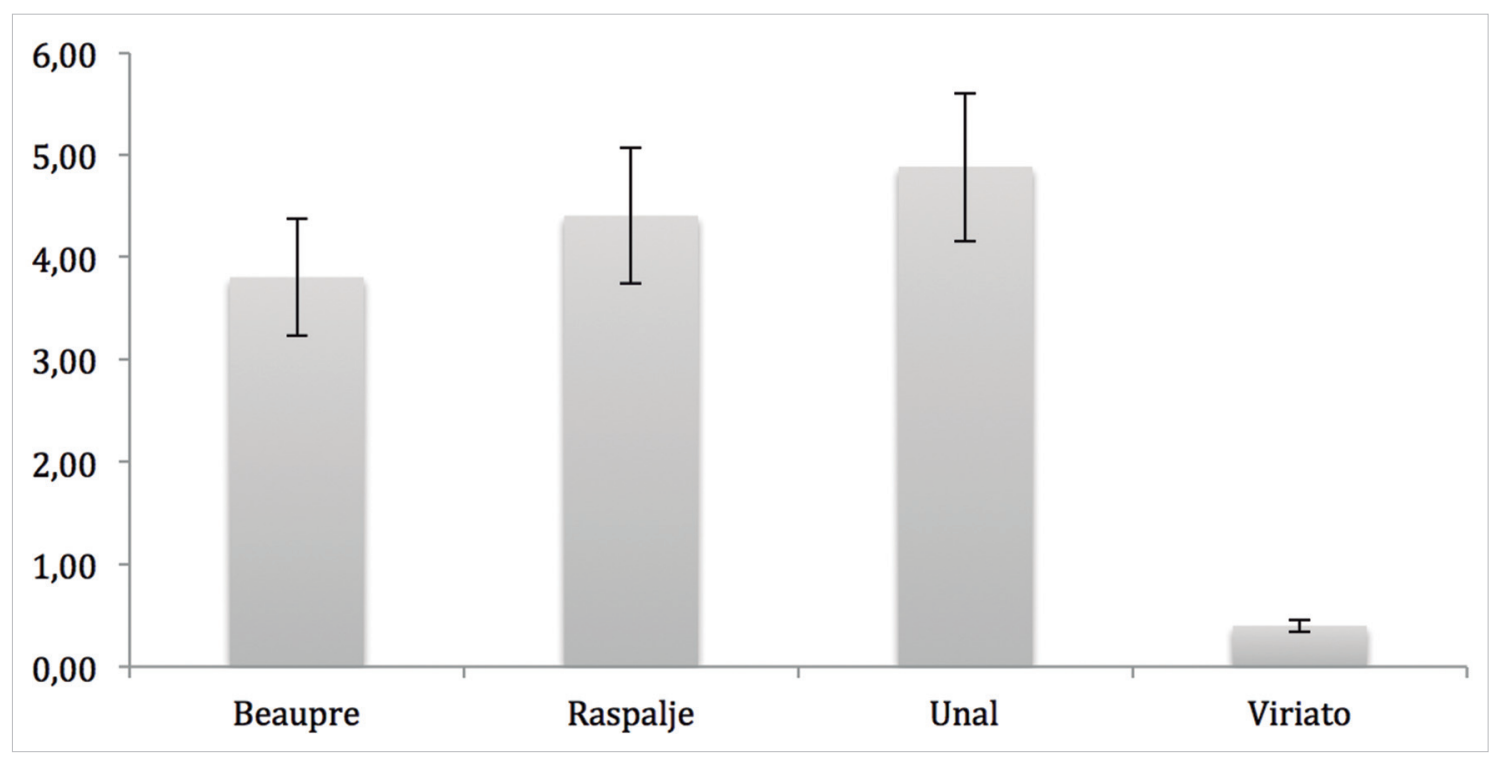

Figura 3. Número medio de heridas por clon e intervalos LSD (least square diference) al 95\% de probabilidad.

Tabla 4. Test de rangos múltiples para el número de heridas por clon $(n=100)$.

Test de rangos múltiples para el número de heridas por clon $(\mathrm{n}=100)$. Método LSD al $95 \%$ de probabilidad

\begin{tabular}{cccc}
\hline Clon & $\mathrm{n}$ & Media & Grupos homogéneos \\
\hline Viriato & 25 & 0,40 & \\
\hline Beaupre & 25 & 3,80 & $\mathrm{X}$ \\
\hline Raspalje & 25 & 4,40 & $\mathrm{X}$ \\
\hline Unal & 25 & 4,88 & $\mathrm{X}$ \\
\hline
\end{tabular}

\section{Conclusiones}

En este trabajo se ha puesto de manifiesto la necesidad de conocer las características y respuestas de cada uno de los clones de chopo ante los problemas bióticos y abióticos que pueden surgir en plantaciones futuras para obtención de biomasa.

Se ha observado una menor sensibilidad a la clorosis férrica del clon "Viriato".

Se ha comprobado la existencia de diferencias significativas en la sensibilidad de los diferentes clones de chopo utilizados al ataque de Paranthrene tabaniformis, observándose sobre todo una menor sensibilidad del clon "Viriato" con respecto a los otros tres.

De las trampas utilizadas, la trampa tipo delta ha dado mejores resultados para el monitoreo y captura del taladro. 
Sería por tanto conveniente tener en cuenta el clon "Viriato" para futuras plantaciones de biomasa por su menor sensibilidad al taladro y mayor resistencia a la clorosis férrica.

\section{Bibliografía}

Baraza, C.; 2013. Cultivos demostrativos de chopos (Populus spp.) a altas densidades con fines energéticos realizados en Aragón. Primeros datos y resultados. $6^{\text {a }}$ Congreso Forestal Español. Vitoria.

Gutierrez, A.; \& Estirado, F.; 1978. Principales plagas del chopo. Hojas divulgadoras del Ministerio de Agricultura. Publicaciones de Extension Agraria.

Moraal, L.G.; Van Der Kraan, C.; \& Van Der Voet, H.; 1993. Studies on the efficacy of the sex attractant of Paranthrene tabaniformis Rott. (Lep. Sesiidae). Journal of Applied Entomology 116: 364-370.

Sixto, H.; Hernández, M.J.; Barrio, M.; Carrasco, J.; \& CañellaS, I.; 2007. Plantaciones del género Populus para la producción de biomasa con fines energéticos: revisión. Investigación Agraria: Sistemas y Recursos Forestales 16 (3): 277-294.

Sixto, H.; Hernández, M.J.; Ciria, P.; Carrasco, J.E.; \& Cañellas, I.; 2010. Manual de cultivo de Populus spp. para la producción de biomasa con fines energéticos. Monografias INIA. Serie Forestal $\mathrm{n}^{\mathrm{o}} 21$. INIA, Madrid, $60 \mathrm{pp}$.

Sixto, H.; Hernández, M.J.; De Miguel, J.; \& Cañellas, I.; 2013. Red de parcelas de cultivos leñosos en alta densidad y turno corto. Publicaciones del Instituto Nacional de Investigación y Tecnología Agraria y Alimentaria. Madrid, 31pp. 
